\title{
Simulation of Viscous Dissipation on Forced Convection Heat Transfer In Porous Medium
}

\author{
Xingwang $\operatorname{TIAN}^{1,2, a}$, Ping WANG ${ }^{\star 1, b}$, Shiming $X U^{1, c}$, Zhenhua SUN ${ }^{1, d}$ \\ ${ }^{1}$ School of Energy and Power Engineering, Dalian University of Technology, Dalian, China \\ ${ }^{2}$ School of Ocean and Civil Engineering, Dalian Ocean University, Dalian, China \\ atxw-1203@126.com, bwp2006@dlut.edu.cn, xsming@dlut.edu.cn, ddlutzhenhuasun@163.com
}

Keywords: Porous media; Convection heat transfer; Viscous dissipation; Power-Law fluid; Numerical simulation

\begin{abstract}
Based on Darcy-Brinkman-Forchheimer flow model, this study analyzed the viscous dissipation effect on the forced, fully developed convection heat transfer in the flat channel filled with saturated porous medium of power-law fluid. Moreover, it derived the dimensionless calculation formulas for the axial velocity distribution and temperature distribution. Then the dimensionless formulas were numerically simulated by using classical Runge-Kutta method under constant heat flux boundary condition. The simulation results show that the dimensionless temperature distribution were affected greatly by Brinkman Number Br, Darcy number Da, Integrated Inertial Parameter F, and power-law index n.
\end{abstract}

\section{Introduction}

The flow and heat transfer in porous media is of great engineering application background [1] and has become a very active research field recently. In the study of convection heat transfer in porous media, the effect of viscous dissipation on the temperature distribution and convection heat transfer characteristics has been widely concerned. The researches of Nield et al.[2,3], Haji-Sheikh et al.[4,5], Hooman et al.[6,7], Hung and Tso [8,9] and Chen and Tso [10,11] et al. have showed that the viscous dissipation greatly affected the convection heat transfer in porous media. However, the studies above merely used the Newton fluid for research, while pay less attention to nonNewton fluid and the viscous dissipation in the energy equation. Limited studies such as Chen and Tso [11] analyzed the influences of the viscous dissipation on forced convection heat transfer in the saturated porous medium channel of power-law fluid. Moreover, they compared the forced convection heat transfer with that of Newton fluid while neglected the influences of inertia effect.

In the studies above, aiming at the problems of "Brinkman-Brinkman" equation [12], the viscous dissipation model of energy equation mainly included endogenous heat dissipation and frictional heat dissipation. The endogenous heat dissipation is generally considered to be the work made by forcing the fluid flowing through porous clearance and depends on flow velocity. The frictional heat dissipation is still controversial: Nield [13] proposed a model that viscous dissipation was equal to the work made by drag force; on the basis of pure fluid N-S equation, Al-Hadhrami [14] deduced a viscous dissipation match term, namely, the frictional heat was treated as an additional term formed by the heat dissipation generated by viscous force, and frictional heat dissipation depends on the shear strain rate.

Based on the analysis of the problems above, this study employed the Darcy-BrinkmanForchheimer flow model and considered the viscous dissipation model proposed by Al-Hadhrami [14] into the energy equation to solve the problems of power-law fluid. Using classical forth-order Runge-Kutta method, the influences of Brinkman Number Br, Darcy number Da and Integrated Inertial parameter $\mathrm{F}$ on the dimensionless temperature distribution of the fluids bearing different rheological indexes was numerically simulated under constant heat flux boundary condition. It was expected that the results obtained can provide certain theoretical basis for the study and application 
of non-Newtonian fluid in engineering field of oil thermal recovery, environmental protection, chemical engineering and so on.

\section{Physical and Mathematical Model}

As shown in Figure 1, this study mainly investigated the convection heat transfer in a flat channel of height $2 \mathrm{H}$ filled with isotropic porous media. Moreover, constant heat flux boundary condition was applied to the upper and lower flat plate surfaces. The flow was steady and full developed, the fluid was the incompressible power-law fluid, and the porous skeleton was rigid.

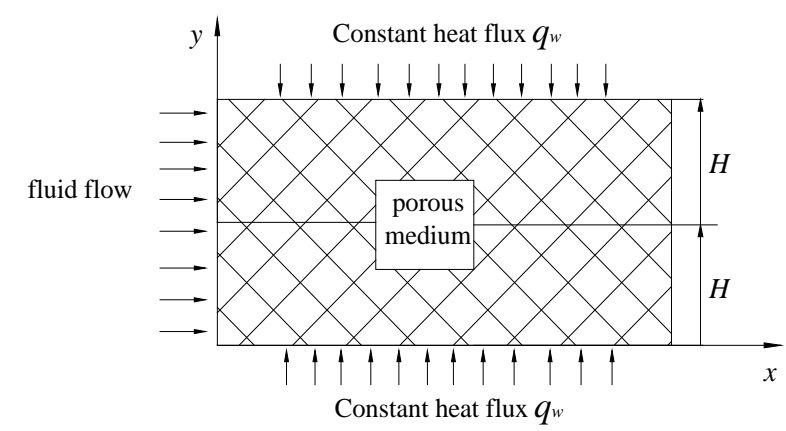

Fig.1 Schematic Diagram of Flow Model in a Flat Channel Filled with Porous Media

The momentum equation of the modified Darcy-Brinkman-Forchheimer flow model is:

$$
\frac{\mu_{e f f}}{\phi^{n}} \frac{d}{d y}\left[\left.\frac{d u}{d y}\right|^{n-1} \frac{d u}{d y}\right]-\frac{\mu}{K^{*}} u^{n}-\rho b u^{2}+\frac{d p}{d x}=0
$$

Where, $u$ is the Darcy flow velocity based on porous medium structure, $m \cdot s^{-1} ; \mu_{\text {eff }}$ is the effective viscosity coefficient of power-law fluid, $N \cdot s \cdot m^{-2} ; \mu$ is the consistency coefficient of power-law fluid, $N \cdot s \cdot \mathrm{m}^{-2} ; \phi$ is the porosity of porous medium; $n$ is a power-law index; $K^{*}$ is the modified permeability of porous medium, $m^{n+1} ; b$ is Forchheimer inertial coefficient, $m^{-1} ; d p / d x$ is pressure gradient. it is a fixed value with unit of $\mathrm{kg} \cdot \mathrm{m}^{-2} \cdot \mathrm{s}^{-2}$.

The definitions as follows: dimensionless coordinate $Y=y / H$; dimensionless viscosity ratio, $M=\mu_{\text {ff }} / \mu$; characteristic reference speed $u_{d}=\left(K^{*} d p /(\mu d x)\right)^{1 / n}$; the dimensionless flow velocity based on reference characteristic speed, $U=u / u_{d}$; the Forchheimer inertial parameters based on reference characteristic velocity: $F=\rho b K^{*}\left(u_{d}\right)^{2-n} / \mu$; the modified Darcy number, $D a=\left(K^{*} / \phi^{n}\right)^{2 /(n+1)} / H^{2}$.

By substituting the dimensionless parameters above into equation (1), the dimensionless momentum equation is:

$$
M \frac{d}{d Y}\left[\left|\frac{d U}{d Y}\right|^{n-1} \frac{d U}{d Y}\right]=\frac{U^{n}+F U^{2}-1}{D a^{(1+n) / 2}}
$$

Corresponding dimensionless boundary condition are expressed as:

$$
\begin{aligned}
& Y=0, \quad U=0 \\
& Y=1, \quad d U / d Y=0
\end{aligned}
$$

Basing on the viscous dissipation model [14], the energy equation can be given by:

$$
\begin{gathered}
k_{\text {eff }} \frac{\partial^{2} T}{\partial y^{2}}+\Omega=\rho c_{P} u \frac{\partial T}{\partial x} \\
\Omega=\frac{\mu u^{n+1}}{K^{*}}+\frac{\mu_{e f f}}{\varphi^{n}}\left|\frac{d u}{d y}\right|^{n-1}\left(\frac{d u}{d y}\right)^{2}
\end{gathered}
$$


Where, $T$ is fluid temperature, $K$; $k_{\text {eff }}$ is the effective thermal conductivity of power-law fluid, $W \cdot \mathrm{m}^{-1} \cdot K^{-1} ; c_{p}$ is the constant-pressure specific heat of power-law fluid $\left(\mathrm{J} \cdot \mathrm{kg}^{-1} \cdot \mathrm{K}^{-1}\right)$; $\Omega$ is the viscous dissipation term of power-law fluid, $\left(W \cdot \mathrm{m}^{-3}\right)$. It includes two terms of endogenous heat dissipation and frictional heat dissipation.

Under a fully developed heat transfer process with constant heat flux boundary condition, the axial heat conduction is negligible. the axial thermal gradient can be simplified into a constant: $\partial T / \partial x=\partial T_{w} / \partial x=\partial T_{m} / \partial x=$ const ; where, $T_{w}$ is the temperature of the plate surface getting contact with porous medium, $K$; The average temperature of power-law fluid $T_{m}=\int_{0}^{H} u T d y / H u_{m}, K$; the average velocity of power-law fluid $u_{m}=\int_{0}^{H} u d y / H, m \cdot s^{-1}$.

As shown in Figure 1, the velocity field and temperature field is symmetric about centreline $\mathrm{y}=\mathrm{H}$. A thin infinitesimal slice is taken along the flow direction of the fluid. According to the first law of thermodynamics, there is:

$$
\frac{d T_{m}}{d x}=\frac{q_{w}+\int_{0}^{H} \Omega}{\rho c H u_{m}}
$$

Where, $q_{w}$ is the heat flux applied by plate surface. The heat flux is positive during the heating process and is negative during the cooling process. It is expressed as:

$$
q_{w}=-\left.k_{\text {eff }} \frac{\partial T}{\partial y}\right|_{y=0}
$$

Definitions are further given: dimensionless temperature $\theta=k_{\text {eff }}\left(T-T_{w}\right) /\left(q_{w} H\right)$; the dimensionless velocity based on average velocity: $U^{\prime}=u / u_{m}$; dimensionless heat conduction ratio $R_{k}=k_{\text {eff }} / k_{f} ; k_{f}$ is the thermal conductivity of power-law fluid, $W \cdot m^{-1} \cdot K^{-1}$; the dimensionless number characterizing viscous dissipation strength: $B r=\mu u_{m}{ }^{n+1} H /\left(q_{w} K^{*}\right)$; the dimensionless number of the modified viscous dissipation strength $B r^{\prime}=B r D a^{(n+1) / 2}=\mu u_{m}{ }^{n+1} /\left(\phi^{n} q_{w} H^{n}\right)$; by substituting equations (5), (6), (7) into energy equation (4) and integration over the cross section of flat channel, the dimensionless energy equation can be obtained:

$$
\begin{aligned}
& \frac{\partial^{2} \theta}{\partial Y^{2}}=U^{\prime}\left[1+B r \int_{0}^{1}\left(U^{\prime}\right)^{n+1} d Y\right. \\
& \left.+M B r^{\prime} \int_{0}^{1}\left|\frac{d U^{\prime}}{d Y}\right|^{n-1}\left(\frac{d U^{\prime}}{d Y}\right)^{2} d Y\right] \\
& -B r\left(U^{\prime}\right)^{n+1}-M B r^{\prime}\left|\frac{d U^{\prime}}{d Y}\right|^{n-1}\left(\frac{d U^{\prime}}{d Y}\right)^{2}
\end{aligned}
$$

Corresponding dimensionless boundary condition are:

$$
\begin{array}{ll}
Y=0, & \theta=0 \\
Y=1, & \partial \theta / d Y=0
\end{array}
$$

The dimensionless Nusselt number is:

$$
N u=\frac{2 H q_{w}}{k_{f}\left(T_{w}-T_{m}\right)}=R_{k} \frac{2 H q_{w}}{k_{e f f}\left(T_{w}-T_{m}\right)}
$$

\section{Numerical Solution Procedure}

According to eq. (2) and eq. (8), it can be deduced that the convection heat transfer of the nonDarcy flow of power-law fluid is intensively nonlinear in porous media. It is difficult to obtain the analytic solution. Therefore, a numerical solution is necessary to describle the physics of the problem. Firstly, the eq. (2) and eq. (8) are recombined to first-order differential equations (11). 
Subsequently, by setting initial value and proper step length, we conduct variables-alternating numerical iteration in range of 0 to 1 using the classical four order Runge-Kutta method until reaching to the requirements of iterative accuracy $\left(10^{-5}\right)$ and boundary condition equation (12).

$$
\left\{\begin{array}{l}
y_{1}^{\prime}=\left(y_{2}\right)^{\frac{1}{n}} \\
y_{2}^{\prime}=\left(\left(y_{1}\right)^{n}+F\left(y_{1}\right)^{2}-1\right) / D a^{\frac{n+1}{2}} / M \\
y_{3}^{\prime}=y_{1} \\
y_{4}^{\prime}=\left(y_{1}\right)^{n+1} \\
y_{5}^{\prime}=\left(y_{2}\right)^{\frac{n+1}{n}} \\
y_{6}^{\prime}=y_{7}^{\prime}=y_{8}^{\prime}=y_{9}^{\prime}=0 \\
y_{10}^{\prime}=y_{11} \\
y_{11}^{\prime}=\frac{y_{1}}{y_{6}}\left[1+B r \frac{y_{8}}{y_{7}}+M B r^{\prime} \frac{y_{9}}{y_{7}}\right] \\
-B r \frac{\left(y_{1}\right)^{n+1}}{y_{7}}+M B r^{\prime} \frac{\left(y_{2}\right)^{\frac{n+1}{n}}}{y_{7}}
\end{array}\right.
$$

Where, $y_{1}=U(Y) ; y_{2}=(d U / d Y)^{n} ; y_{3}=\int_{0}^{Y} U d Y ; y_{4}=\int_{0}^{Y} U^{n+1} d Y ; y_{5}=\int_{0}^{Y}(d U / d Y)^{n+1} d Y ; y_{6}=U_{m}$; $y_{7}=\left(U_{m}\right)^{n+1} ; y_{8}=\int_{0}^{1} U^{n+1} d Y ; y_{9}=\int_{0}^{1}(d U / d Y)^{n+1} d Y ; y_{10}=\theta(Y) ; y_{11}=d U / d Y$

First-order equation (11) should satisfy the following boundary conditions:

$$
\left\{\begin{array}{l}
y_{1}(0)=y_{2}(1)=y_{3}(0)=y_{4}(0) \\
=y_{5}(0)=y_{10}(0)=y_{11}(1)=0 \\
y_{6}(0)=y_{3}(1) \\
y_{7}(0)=\left(y_{3}(1)\right)^{n+1} \\
y_{8}(0)=y_{4}(1) \\
y_{9}(0)=y_{5}(1)
\end{array}\right.
$$

After solving the velocity distribution and temperature distribution, the expression of Nusselt number is getable through equation (10).

$$
N u=-2 R_{k} y_{6} / \int_{0}^{1} y_{1} y_{10} d Y
$$

To validate the validity of numerical scheme, the results obtained are compared with the results of reference [6] when $M=R_{k}=1$ andBr=0, For Newton fluid $(n=1)$, in the case of small Darcy number $(\mathrm{Da} \leqslant 0.001)$, one finds $\mathrm{Nu}=5.92$, the results approximates $\mathrm{Nu}=6$ for the case of Darcy flow. For the case of large Darcy number $(\mathrm{Da} \geqslant 1000)$, one finds $\mathrm{Nu}=4.121$ approaching to 70/17 for the plane Poisueille flow limit

\section{Results and Discussion}

The main influencing factors of dimen-sionless temperature distribution as follows: 


\subsection{Brinkman Number}

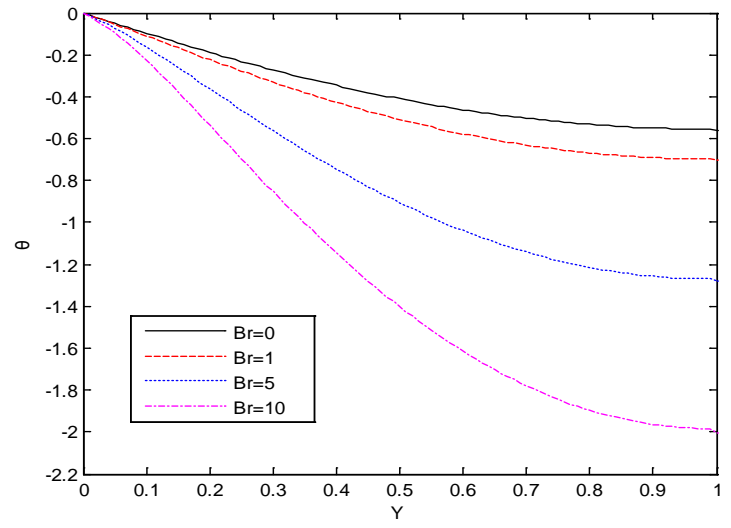

(a) $n=0.5$

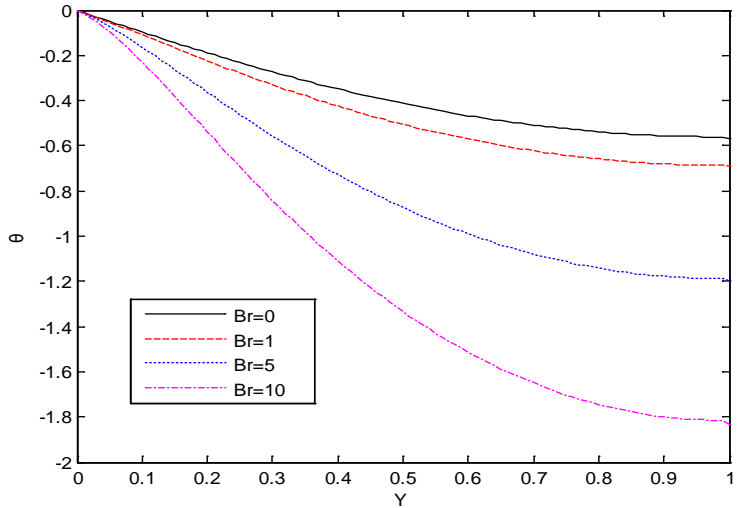

(b) $\mathbf{n}=\mathbf{1 . 0}$

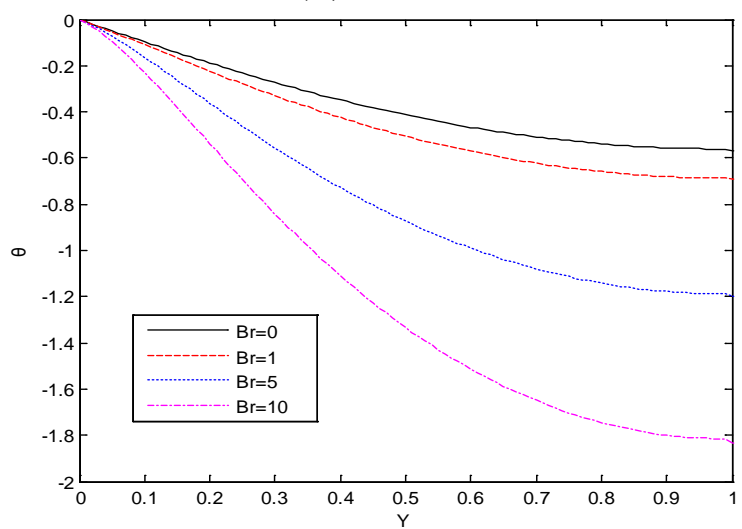

(c) $n=1.5$

Fig.2 The Effect of Brinkman Number on the Dimensionless Temperature Distribution

Figure 2 shows the variations of dimensionless temperature with $\mathrm{Br}$ under the influences of power-law index $\mathrm{n}$ when $\mathrm{M}=1, \mathrm{Da}=0.1$ and $\mathrm{F}=1$. As shown in this figure, when $\mathrm{Br}=0$, the dimensionless temperature reduces toward the channel center gently. In addition, the temperature distribution profiles of the three fluids are close to each other. As $\mathrm{Br}$ increase, dimensionless temperature decreases rapidly. Meanwhile, the shear thinning fluid displays larger variations than the other two fluids due to the high velocity gradient (large convection heat transfer rate). However, the higher frictional thermal viscous dissipation generated on the region near the plate surface reduces the effective convection heat transfer rate at high $\mathrm{Br}$ value. As a result, the shear thinning fluid is greatly influenced by the viscous dissipation. 


\subsection{Darcy Number}

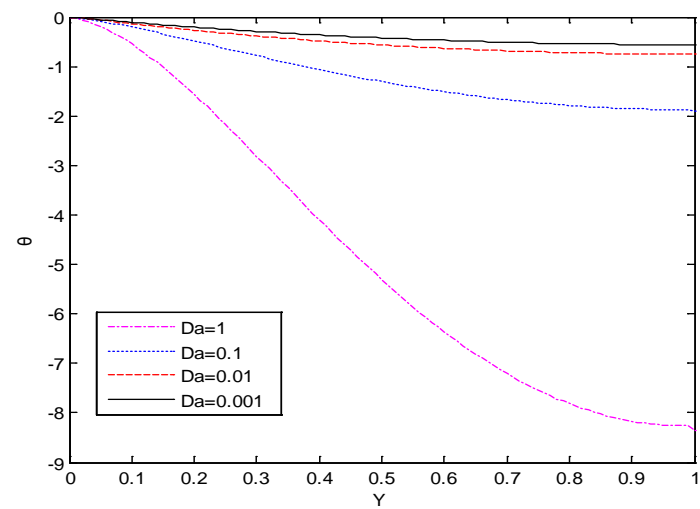

(a) $\mathbf{n}=0.5$

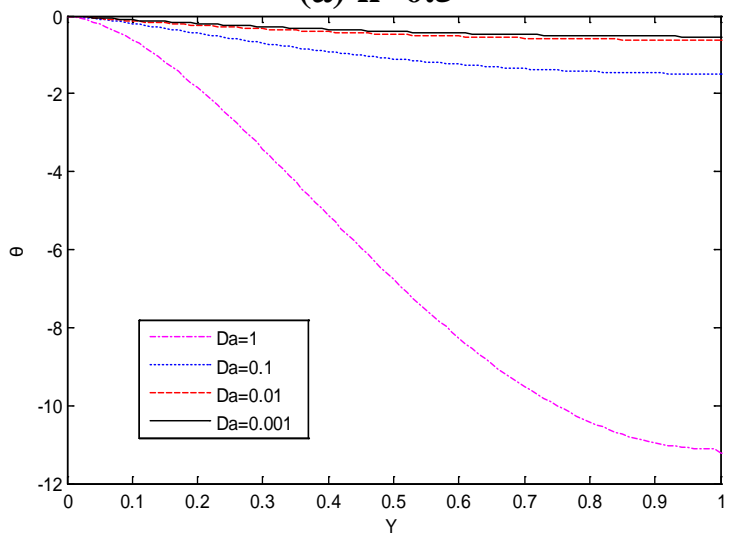

(b) $\mathbf{n}=\mathbf{1 . 0}$

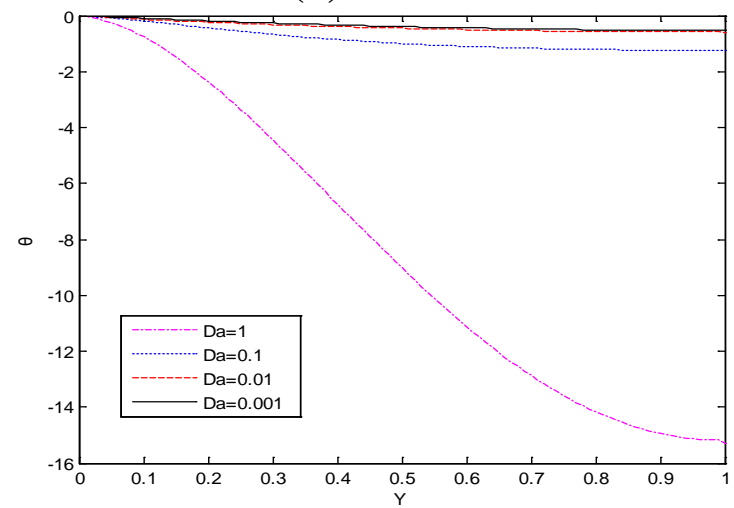

(c) $\mathrm{n}=1.5$

Fig.3 The Effect of Darcy Number on the Dimensionless Temperature Distribution

Figure 3 illustrates the variations of dimensionless temperature with Da under the influences of power-law index $\mathrm{n}$ when $\mathrm{M}=1, \mathrm{Br}=10$ and $\mathrm{F}=0$. It is observed that the dimensionless temperature reduces toward the channel center gently for small Da number. The temperature of shear thickening fluid maximizes attributing to the large velocity gradient of shear thickening fluid (large convection heat transfer rate). More frictional thermal viscous dissipation generated in the region near the plate surface reduces the convection heat transfer rate. Nevertheless, in the case of small Da number, the dominant endogenous heat viscous dissipation overcomes the frictional thermal viscous dissipation and suffers less influences from viscous dissipation.

At the same time, dimensionless temperature decreased rapidly when given large Da. Moreover, as Da increase, the shear thickening fluid presents more obvious variations than the other fluids. This is because of that the shear thickening fluid is subjected to great influences $(n=1.5)$ of Da. On one hand, larger Da enables more frictional thermal viscous dissipation being generated on the region near the plate surface and thus reduces the convection heat transfer rate; on the other hand, 
owning to the small velocity gradient (small convection heat transfer rate), shear thickening fluid is greatly influenced by the viscous dissipation.

\subsection{Integrated Inertial Parameter}

Figure 4 is a description of the variations of dimensionless temperature distribution with $\mathrm{F}$ under the influences of power-law index $n$ when $M=1, B r=10$, and $\mathrm{Da}=0.1$. As illustrated in Fig.4, the shear thinning fluid reduces more significantly than the other two fluids because of the high velocity gradient. The more frictional thermal viscous dissipation generated declines the convection heat transfer rate. In addition, shear thinning fluid shows smaller velocity gradient variation ranges in three fluids and is less influenced by the viscous dissipation. In contrast, shear thickening fluid suffers from more influences of viscous dissipation owning to the large variations of velocity gradient.

Moreover, as $\mathrm{F}$ increase, the dimensionless temperatures of the three fluids all sharply decrease toward the channel center. The shear thinning fluid and shear thickening fluid show minimum and maximum temperature variations respectively. In case of neglecting viscous dissipation, the velocity gradient increase of the fluid near the plate surface produces stronger convection heat transfer as increasing $\mathrm{F}$, resulting in the temperature rise rather than temperature reduction. However, if viscous dissipation is taken into consideration, the situation is just the reverse. The increase of $\mathrm{F}$ strengthens the velocity gradient of the fluid near the plate surface, leading to the increase of the frictional thermal viscous dissipation generated. The increased frictional thermal viscous dissipation reduces the overall convection heat transfer rate and the dimensionless temperature finally.

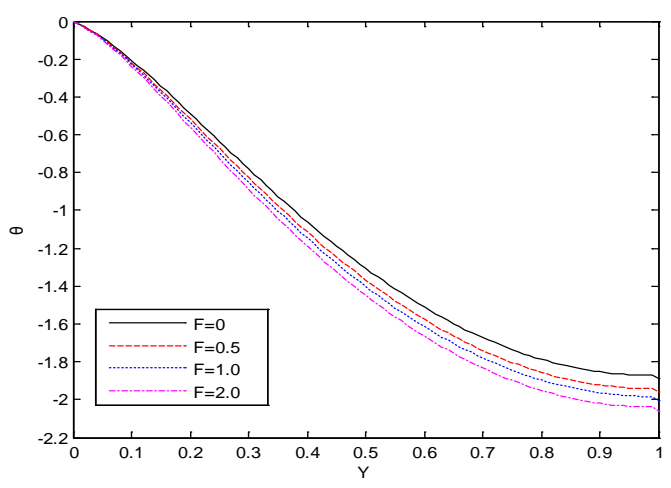

(a) $n=0.5$

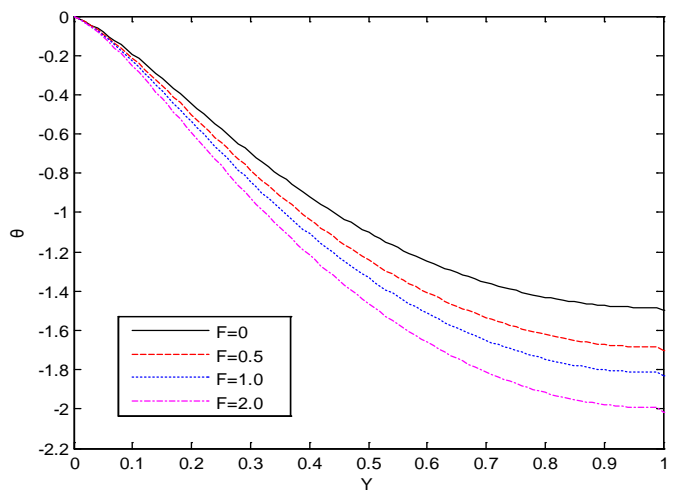

(b) $n=1.0$ 


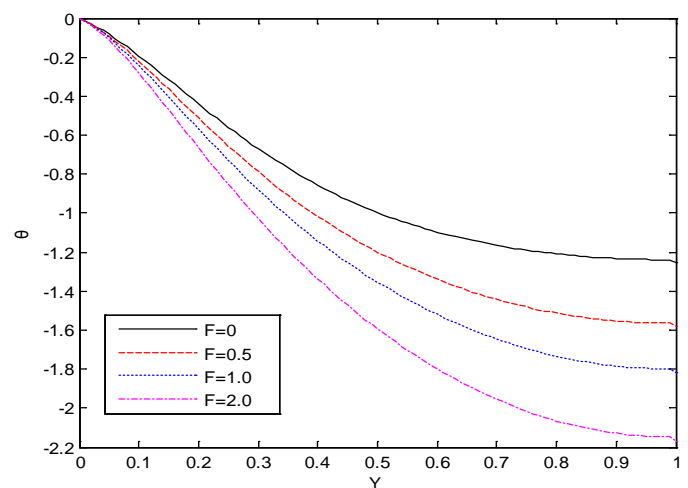

(c) $\mathrm{n}=1.5$

Fig.4 The Effect of the Integrated Inertial Parameter on the Dimensionless Temperature Distribution

\section{Conclusion}

The study investigated the convection heat transfer of power-law fluid in the flat plate channel filled with porous media based on Darcy-Brinkman-Forchheimer flow model. It is observed with increasing $\mathrm{Br}$ or more intense viscous dissipation, the dimensionless temperature of power-law fluid reduced sharply, resulting in the reduction of the convection heat transfer rate. The results suggest that viscous dissipation greatly affect the temperature distribution and should not be neglected. The temperature distribution of fluid was also subjected to the influences of dimensionless parameters, such as Darcy number, integrated inertial parameter, and power law index.

\section{Acknowledgements}

Project supported by the National Natural Science Foundation of China (Grant No.51276029)

\section{References}

[1] Nield, D.A., Bejan, A., Convection in Porous Media $3^{\text {rd }}$ ed.. Springer-Verlag: New York, 2006.

[2] Nield, D.A., Kuznetsov, A.V., Xiong, M., "Thermally developing forced convection in a porous medium: parallel plate channel with walls at uniform temperature, with axial conduction and viscous dissipation effects”, International Journal of Heat and Mass Transfer, vol. 46, pp. 64351, 2003.

[3] Nield, D.A., Kuznetsov, A.V., Xiong, M., "Effects of viscous dissipation and flow work on forced convection in a channel filled by a saturated porous medium", Transport Porous Media, vol. 56, pp. 351-67, 2004.

[4] Haji-Sheikh, A., Minkowycz, W.J., Sparrow, E.M., “Green’s function solution of temperature field for flow in porous passages”, International Journal of Heat and Mass Transfer vol. 47, pp. 4685-95, 2004.

[5] Haji-Sheikh, A., Nield, D.A., Hooman, K., "Heat transfer in the thermal entrance region for flow through rectangular porous passages”, International Journal of Heat and Mass Transfer, vol. 49, pp.3004-15, 2005.

[6] Hooman, K., Gurgenci, H., "Effects of viscous dissipation and boundary conditions on forced convection in a channel occupied by a saturated porous medium", Transport Porous Media, vol. 68, pp.301-19, 2007.

[7] Hooman, K., Haji-Sheikh, A., Nield, D.A., “Thermally developing Brinkman-Brinkman forced convection in rectangular ducts with isothermal walls”, International Journal of Heat and Mass Transfer, vol. 50, pp. 3521-33, 2007.

[8] Y.-M. Hung, C.P. Tso., "Temperature variations of forced convection in porous media for heating and cooling processes: internal heating effect of viscous dissipation”, Transport Porous Media, vol. 75, pp. 319-32, 2008. 
[9] Y.-M. Hung, C.P. Tso., "Effects of viscous dissipation on fully developed forced convection in porous media”, International Communications in Heat and Mass Transfer, vol. 36, pp. 597-603, 2009.

[10] G.M. Chen, C.P. Tso., "Forced convection with viscous dissipation using a two equation model in a channel filled by a porous medium”, International Journal of Heat and Mass Transfer, vol. 5, pp. 1791-1804, 2011.

[11] G.M. Chen, C.P. Tso., "Effects of viscous dissipation on forced convective heat transfer in a channel embedded in a power-law fluid saturated porous medium”, International Communications in Heat and Mass Transfer , vol. 38, pp.57-62, 2011.

[12] Nield, D.A., “A note on a Brinkman-Brinkman forced convection problem”, Transport Porous Media, vol. 64, pp. 185-8, 2006.

[13] Nield, D.A., "Resolution of a paradox involving viscous dissipation and nonlinear drag in a porous medium”, Transport Porous Media, vol 41, pp. 349-57, 2000.

[14] A.K. Al-Hadhrami, L. Elliot, D.B. Ingham., "A new model for viscous dissipation in porous media across a range of permeability values", Transport Porous Media, vol. 53, pp. 117-22, 2003. 